Original scientific paper

\title{
CONGESTION RISK PROPAGATION MODEL BASED ON MULTI-LAYER TIME-VARYING NETWORK
}

\author{
Huang, J. H.; Sun, M. G. \& Cheng, Q.\# \\ School of Economics and Management, Fuzhou University, Fuzhou, 350116, China \\ E-Mail: chengquan@fzu.edu.cn $\left({ }^{\#}\right.$ Corresponding author $)$
}

\begin{abstract}
To quantify the responses of drivers to traffic information and the congestion evacuation effect on the basis of traffic guidance information, a multilayer network congestion risk propagation model of urban roads was built to analyse the influence of the advanced traveller information system (ATIS) penetration rate, group behaviours of drivers, and traveller flow distribution features on the traffic congestion risk propagation of urban roads. Meanwhile, the dynamic evolutionary characteristics of group behaviours of drivers in a road network under the guidance of traffic information were analysed with the microscopic Markov chain approach (MMCA). A simulation analysis of the artery network in the fourth ring of Beijing was also carried out. Results demonstrated that the influence of traffic information and drivers' information reinforcement psychology on congestion risk propagation depend on the aggregation effect caused by traffic information. Increasing the ATIS market penetration rate and drivers' acceptance of information is beneficial to relieve traffic congestion as long as the drivers' aggregation effect is within a critical range.

(Received in August 2021, accepted in November 2021. This paper was with the authors 5 weeks for 1 revision.)
\end{abstract}

Key Words: Urban Traffic, Congestion Propagation Analysis, Microscopic Markov Chain, Traffic Information, Group Behavioural Characteristics of Drivers

\section{INTRODUCTION}

An advanced traveller information system (ATIS) can solve traffic congestion problems caused by unreasonable road network design and uneven traffic flow distribution by issuing real-time congestion warning information and dynamic route guidance. Compared with the traditional traffic control mode, traffic information guidance is not a compulsory measure. Its effect on congestion control is not only determined by the road network structure and urban traffic needs, but is also closely related to the drivers' acceptance and utilization of information. Therefore, examining the route choice behaviours of drivers under the guidance of traffic information has important significance for exploring congestion control means and improving the congestion control effect.

Recent theoretical studies on the influence of traffic information issued by ATIS on traffic congestion focus on traffic information issuing characteristics, driver attributes, and drivers' attitude toward traffic information [1]. Many research streams have been derived, such as the ATIS market penetration rate [2-4], validity and reliability of the issued information $[5,6]$, route choice behavioural process of drivers [7], and the drivers' acceptance and overreaction to traffic information $[8,9]$. Some studies have examined the influence of the route choice gaming of driver groups on traffic congestion propagation [10-12]. However, the major research direction was biased toward the exploration of individual driver behaviour. Studies on the influence of the evolution from the individual behaviours of drivers to group behaviours on traffic congestion are rare. Moreover, most of the selected factors that can influence the route choice behaviours of drivers are objective indexes. The subjective indexes, such as the drivers' attitude toward traffic information, are ignored. With respect to research methods, existing studies are mainly qualitative, and quantitative analysis of the relationship between traffic congestion propagation and the applications of information technology (e.g., ATIS) are lacking. Simulating the complicated large-scale real-time traffic flow changes under the influence of 
traffic information, the route choice behavioural characteristics of drivers, and road network characteristics is an important issue that must be solved urgently.

In this study, drivers' responses to traffic guidance information are chosen as the research objects. Analysing the influence of traffic guidance information on the behaviours of drivers and its validity can provide traffic administration with references for improving traffic information issuing strategies. With references to the traffic congestion and warning information interaction model [13-15] and the research outcomes from the coupling propagation dynamic model based on multilayer network [16-19], this study makes the following innovations. First, a multilayer time-varying network coupling model is built. The route choice behaviours of driver groups on the microscopic layer are mapped onto the traffic network and their influences on the traffic flow evolution on the road network are analysed. Second, the influences of the ATIS penetration rate, traffic information warning, and the reinforcement and aggregation effects of drivers on traffic congestion risk propagation are discussed. The relationships between different traffic guidance information is suing strategies and the traffic congestion control effect are analysed.

\section{STATE OF THE ART}

How traffic information issuing influences the traveling efficiency of the road network has been one of the key problems in the urban traffic domain. The research contents of existing literature involve the following aspects:

The effects study of ATIS information on traffic congestion. Studies on the ATIS market penetration rate that the individual local actions of drivers might cause worse consequences to themselves and the entire traffic system if they cannot receive the traffic information. If the proportion of drivers who receive information ranges between 0.25 and 0.5 , then the performances of the entire traffic system will be improved [2]. However, when the proportion of drivers receiving traffic information exceeds a certain value, the individual local actions of drivers might cause new congestions under the influence of the information, thereby decreasing the operation performances of the traffic system [3]. When numerous drivers are both information providers and information users, the system performance declines [14]. As a result, providing information to some drivers rather than to all drivers can optimize the traffic flow and better relieve traffic congestion. Equipping vehicles with driver information systems beyond the critical numerical range is unnecessary [4]. With respect to studies on compliance rate to guidance, not all drivers may follow the suggestions of ATIS equipment despite their high accuracy, and this circumstance is related to the bounded rationality and cognition of humans [5]. Drivers only change their choices when the changes of the traffic system or traveling characteristics (e.g., traveling time) are greater than the threshold of individual situations [6].

Route choice behavioural characteristics study of drivers. Studies on drivers' responses to ATIS information deepen continuously from the individual behavioural mechanism of drivers to their social choice behavioural mechanism. Dai et al. [7] proposed a dynamic procedure of daily route choice behaviours of commuters in a car networking environment and described the microscopic and macroscopic behaviours of drivers-vehicles-unit agency by the multi-agent simulation method. With considerations to cost estimation and route choice of drivers' behaviours, Diop et al. [8] confirmed that the perception of information quality, attitude, and familiarity to route transfer had direct or indirect influences on route change behaviours. Specifically, attitude toward and intention of redirection had significant impacts on route transfer behaviours. Khoo and Asitha [9] established that traveling choice is related to the perceived congestion degree of drivers and that the possibility of route changing increases with the congestion degree. When the traffic flow rate on the available substitutive route exceeds the 
critical value and causes queuing up or "congestion transfer," the benefits of the guided vehicles decline accordingly [10]. Klein and Ben-Elia [11] explored the influence of an ATIS according to the system optimization on individual route choice and the follow-up mass traffic condition. In the experiment, ATIS provided information to participants through external incentives of route recommendation and route following on the basis of system optimization distribution, and participants adapted to the new information coping strategies in four ways. The mechanism by which the implicit cooperation among self-interested participants appeared on the simple binary road network was analysed. Yu et al. [12] proposed a dual dynamic daily flow distribution model on a large-scale network and included the bounded rationality and information sharing mechanism into the macroscopic choice modelling framework. Results emphasized the necessity of modelling the network transient and unbalanced states. In summary, the above studies have simulated the influence of the route choice behaviours of drivers on traffic flow distribution by using many network flow distribution models, such as the network equilibrium theory model, multi-agent model, route gaming model, and driving device simulation. However, most prior research examined the network equilibrium in a simple route network only and have not verified the performances of models in practical large-scale road networks.

This study hypothesizes that a network composed of driver groups is a time-varying group structural network. Additionally, existing studies on the influence of traffic information on traffic congestion propagation mainly emphasized the individual route choice behaviours of drivers, but overlooked the drivers' group behavioural characteristics. As a result, the traffic congestion propagation process in urban roads is chosen as the research object in this article. A multilayer time-varying network unaware aware unaware susceptible infected susceptible (UAU-SIS) model on the basis of driver groups was developed. The model was solved by a microscopic Markov chain approach (MMCA) with consideration to the influence of the ATIS penetration rate and drivers' responses to traffic information on traffic congestion propagation process. Subsequently, the practical traffic flows on urban roads were simulated with PyCharm software. In this simulation, the values of the Beijing Road network matrix and traveller network matrix were determined according to the different ATIS penetration rates as well as the reinforcement and aggregation effects of drivers. Simulation results verified that the MMCA based on multilayer time-varying network model is applicable for the simulation of traffic congestion propagation.

The remainder of this study is organized as follows. Section 3 constructs a model of the influence of the group behaviours of drivers on congestion risk propagation on urban roads and determines the simulation logics of the UAU-SIS model on the research problem of this study. The rules of the MMCA are also established for the research problem. Section 4 solves the threshold of the congestion risk outburst by using the PyCharm software and MMCA. The influence of the ATIS penetration rate as well as the aggregation and reinforcement effects of drivers who receive information on congestion risk propagation are determined and simulated. Section 5 summarizes the conclusions of this article.

\section{METHODOLOGY}

\subsection{Problem description and assumption}

Multilayer time-varying network is a complex network system composed of information subnet, road subnet and trip subnet, as shown in Fig. 1.

Information subnet: the nodes in this subnet refer to individual drivers, and two states occur. The nodes in State U (Unaware) have not gained any information (this state also implies that the nodes have not been persuaded by information). The nodes in State A (Aware) gain traffic information and sides reflect normal communication among nodes. 
Road subnet: road crosses or stations are used as the nodes and practical roads are sides to reflect the propagation relations of congestion risks in the road subnet. Specifically, two states of congestion risk transmission occur at each node, namely, the susceptible (S) and infected (I) states.

Trip subnet: the nodes in this subnet reflect the individual drivers, and the two behaviours involved entail changing routes according to the information tips or staying with the existing route. Both information and behaviour layers are time-varying networks that characterize the dynamic flows of driver groups in the road network.

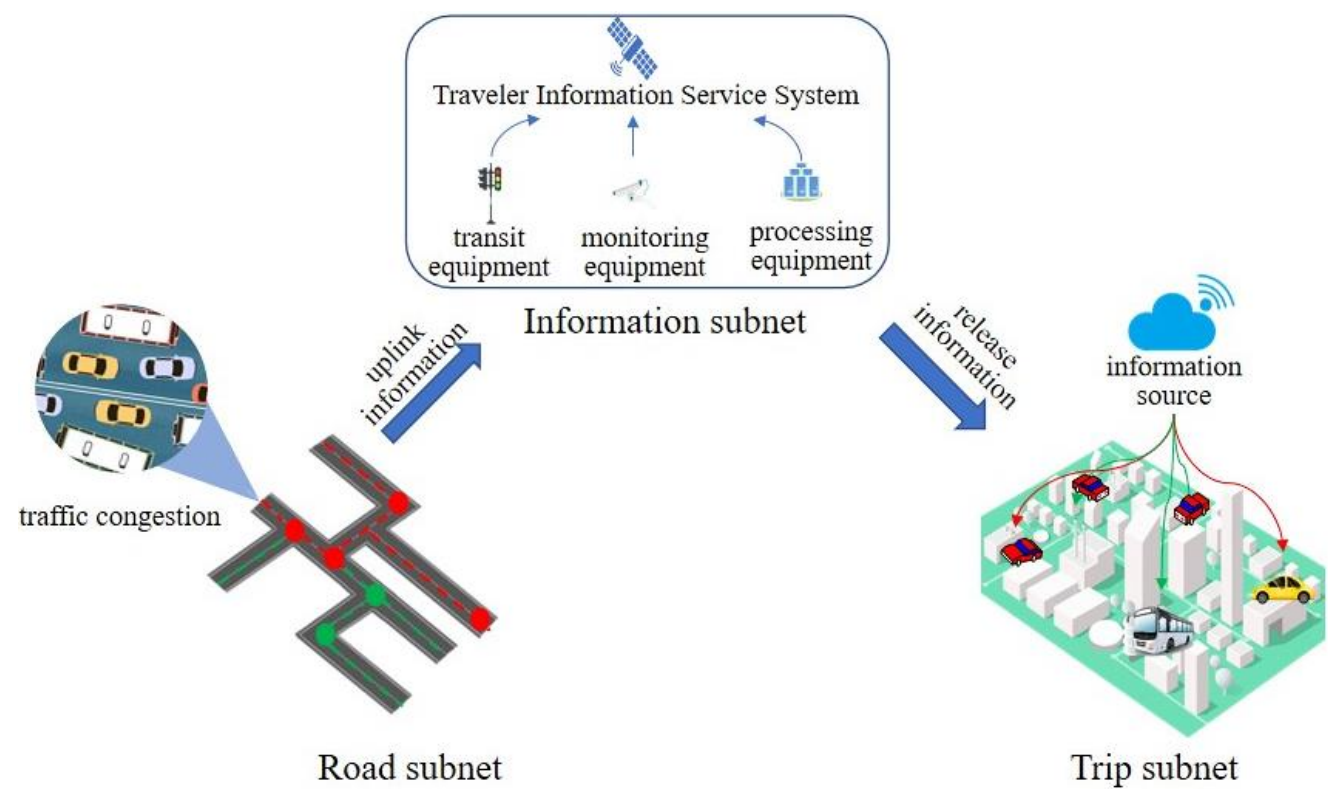

Figure 1: Multi-layer time-varying network UAU-SIS model.

Table I: Description of the notation.

\begin{tabular}{|c|l|}
\hline Parameter & \multicolumn{1}{c|}{ Definitions } \\
\hline$a_{i j}$ & Adjacency matrix of information layer nodes \\
\hline$b_{i j}$ & Adjacency matrix of physical layer nodes \\
\hline$\left\{x_{i}\right\}_{i=1}^{n}$ & $\begin{array}{l}\text { The sequence represents the set of group sizes in the information layer, where } n \text { is the } \\
\text { number of groups }\end{array}$ \\
\hline$X=\left\{x_{i j}\right\}_{i, j=1}^{M}$ & $\begin{array}{l}X \text { represents the group matrix of the information layer, } x_{i j}=1 \text { represents the same group of } \\
\text { two nodes } i \text { and } j \text { in the information layer }\end{array}$ \\
\hline$\beta_{i}{ }^{(\mathrm{U})}$ & Traffic transfer probability of congestion node $i$ without information \\
\hline$\beta_{i}{ }^{\text {A) }}$ & Traffic transfer probability of congestion node $i$ with information \\
\hline$\mu_{i}$ & Recovery rate of congested node $i$ \\
\hline$\delta$ & Information forgetting rate \\
\hline$\lambda_{1}$ & Intra group information diffusion probability \\
\hline$\lambda_{2}$ & Inter group information diffusion probability \\
\hline$\gamma$ & Traffic information warning \\
\hline$r_{i}$ & The probability that a node $i$ without information is not informed in the information layer \\
\hline
\end{tabular}

Given to route choice behaviours of the informed drivers, the commuting choices of uninformed drivers who are trapped in traffic congestions are influenced by traffic information. The aggregation effect refers to the probability of changing routes according to information guidance after driver groups at a road node have confirmed information reliability. The reinforcement effect reflects the memory of individual drivers in relation to the received 
information. It refers to the psychological threshold which indicates that drivers are persuaded by the information. The notation used in the model is shown in Table I.

\subsection{Evolution process of network traffic flow}

The evolution of network traffic flow under the traffic information is abstracted into a Markov process. The kinetic equations of four possible states of the UAU-SIS model are established according to the variant MMCA of the discrete time Markov approach in the complicated network epidemic analysis. In this study, a theoretical analysis of the UAU-SIS model is performed by using MMCA. Node $i$ in the physical layer and the corresponding node in the information layer have four possible coupling states in the sense of probability: (Aware and Infected, AI), (Aware and Susceptible, AS), (Unaware and Infected, UI), and (Unaware and Susceptible, US).
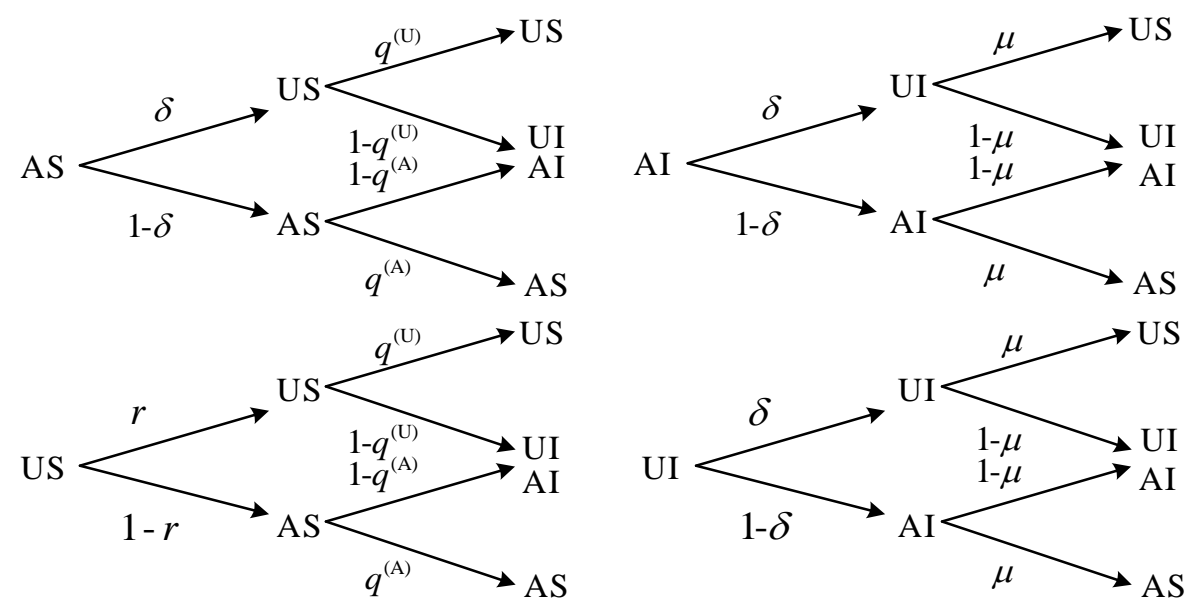

Figure 2: UAU-SIS state transfer tree of the multi-layer time-varying network.

For node $i$ in the physical layer, we suppose that all nodes in the same group of the information layer are $j\left(j=1, \ldots, x_{i}\right)$. Then, the group consensus of these nodes is defined by the mean perception probability: $p_{i}^{A}(t)=\frac{1}{x_{i}} \sum_{j=1}^{x_{i}} p_{i j}^{A}(t)$. These probabilities meet the normalization conditions:

$$
p_{i}^{A I}(t)+p_{i}^{A S}(t)+p_{i}^{U I}(t)+p_{i}^{U S}(t)=1
$$

Suppose $r_{i j}(t)$ reflects the probability that the unknown node $j$ has not entered into the perceived state (node $j$ of the information layer and node $i$ in the physical layer are in the same group).

$$
r_{i j}(t)=\alpha-c_{i j} \prod_{k \in X_{j}}\left[1-a_{j k} p_{k}^{A}(t) \lambda_{1}\right] \times \prod_{k \notin X_{j}}\left[1-a_{j k} p_{k}^{A}(t) \lambda_{2}\right]
$$

$p_{k}^{A}(t)=p_{k}^{A I}(t)+p_{k}^{A S}(t)$, where $\alpha$ refers to the herd effect. In other words, individual drivers have memories of the received information, and the times of information receptions have a cumulative effect on the corresponding behaviours of individuals. The more times of information that an individual receives, the higher the probability of generating the corresponding behaviour. According to the homogeneity of the network, for a given node $i$ in the physical layer, this study used $r_{i}(t)=\frac{1}{x_{i}} \sum_{j=1}^{x_{i}} r_{i j}(t)$ to express the probability that the nodes in the physical layer have not perceived nodes in the information layer.

Suppose $q_{i}^{(\mathrm{A})}(t)$ and $q_{i}^{(\mathrm{U})}(t)$ are the probabilities of no infection of node $i$ when it gains information or not, respectively. These probabilities are defined as follows: 


$$
\begin{aligned}
q_{i}^{(\mathrm{A})}(t) & =\prod_{j}\left(1-b_{j i}\left(p_{j}^{(\mathrm{UI})}(t) \beta_{i}^{(\mathrm{U})}+p_{j}^{(\mathrm{AI})}(t) \beta_{i}^{(\mathrm{A})}\right)\right) \gamma \\
q_{i}^{(\mathrm{U})}(t) & =\prod_{j}\left(1-b_{j i}\left(p_{j}^{(\mathrm{UI})}(t) \beta_{i}^{(\mathrm{U})}+p_{j}^{(\mathrm{AI})}(t) \beta_{i}^{(\mathrm{A})}\right)\right)
\end{aligned}
$$

where $\beta_{i}{ }^{(\mathrm{U})}$ refers to the proportion of drivers who have perceived the congestion but have no information about route transfer. $\beta_{i}{ }^{(\mathrm{A})}$ is the cumulative effect of driver groups caused by traffic information, that is, the proportion of drivers who have perceived the congestion and information and changed routes accordingly. $\gamma$ is the traffic information warning, that is, the proportion of drivers who are not in the congestion state and have the information and are willing to adopt actions in advance to avoid congestion.

Suppose $\mu_{i}(t)$ refers to the recovery probability of the congestion node $i$. It is defined as:

$$
\mu_{i}(t)=\frac{\sum_{j} b_{j i}\left(p_{j}^{(\mathrm{AS})}(t)+p_{j}^{(\mathrm{US})}(t)\right)}{\operatorname{sum}(j)}
$$

The kinetic equation of the entire model according to the state transfer tree in Fig. 2 is as follows:

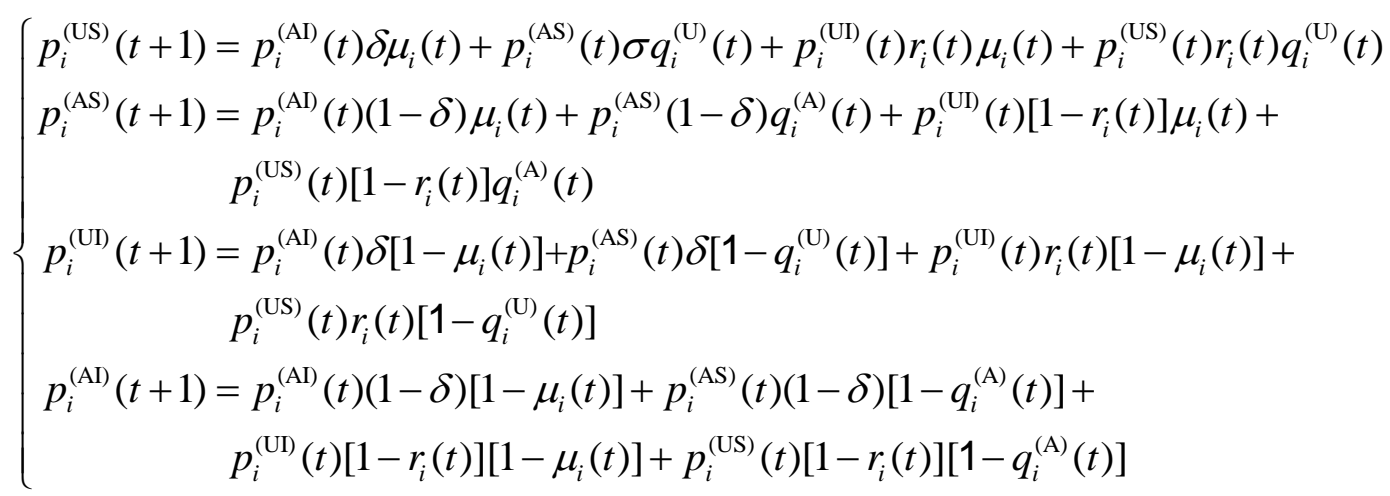

where $t$ is the current time step and $t+1$ is the next time step. In each time step, $p_{i}^{(\mathrm{US})}+p_{i}^{(\mathrm{AS})}+p_{i}^{(\mathrm{AI})}+p_{i}^{(\mathrm{UI})}=1$ to meet normal conditions. When the time step is large enough, the proportions of the node states reach a stable state. When $t+1$ tends to be infinite,

$$
\left\{\begin{array}{l}
p_{i}^{(\mathrm{AI})}(t+1)\left|t \rightarrow \infty=p_{i}^{(\mathrm{AI})}(t)\right| t \rightarrow \infty=p_{i}^{(\mathrm{AI})} \\
p_{i}^{(\mathrm{AS})}(t+1)\left|t \rightarrow \infty=p_{i}^{(\mathrm{AS})}(t)\right| t \rightarrow \infty=p_{i}^{(\mathrm{AS})} \\
p_{i}^{(\mathrm{US})}(t+1)\left|t \rightarrow \infty=p_{i}^{(\mathrm{US})}(t)\right| t \rightarrow \infty=p_{i}^{(\mathrm{US})} \\
p_{i}^{(\mathrm{UI})}(t+1)\left|t \rightarrow \infty=p_{i}^{(\mathrm{UI})}(t)\right| t \rightarrow \infty=p_{i}^{(\mathrm{UI})}
\end{array}\right.
$$

Next, the evolution of congestion risk propagation under any initial state information diffusion can be simulated by combining Eqs. (1) to (7). The steady state of expected infection density $\rho=\frac{1}{\mathrm{~N}} \sum_{i=1}^{N} \rho_{i}^{(\mathrm{I})}=\frac{1}{\mathrm{~N}} \sum_{i=1}^{N}\left(\rho_{i}^{(\mathrm{UI})}+\rho_{i}^{(\mathrm{AI})}\right)$ is solved through iteration, where $\rho_{i}^{(\mathrm{I})}$ is the infection probability of node $i$ in the physical layer.

\subsection{Infection threshold analysis}

Infection threshold is an important parameter that determines whether the risk leads to an outburst or dies eventually. Estimation of the critical infection threshold is the first step to analyse the characteristics of the existing model. Near the outburst threshold, the probability of any infected node is close to 0 . Therefore, we may hypothesize that $p_{i}{ }^{(\mathrm{AI})}(t)=\varepsilon_{1} \ll 1$, $p_{i}{ }^{(\mathrm{UI})}(t)=\varepsilon_{2} \ll 1$, and $p_{i}{ }^{(\mathrm{I})}(t)=p_{i}{ }^{(\mathrm{AI})}(t)+p_{i}{ }^{(\mathrm{UI})}(t)=\varepsilon \ll 1$. According to the above hypothesis and 
formula, $p_{i}{ }^{(\mathrm{US})}(t+1)$ and $p_{i}^{(\mathrm{AS})}(t+1)$ in Eq. (7) can be approximately expressed as $q_{i}^{(\mathrm{A})}(t)$ and $q_{i}{ }^{(\mathrm{U})}(t)$ in Eq. (5) can be approximately expressed as:

$$
\begin{aligned}
q_{i}^{(\mathrm{A})}(t) & =\prod_{j}\left(1-b_{j i}\left(p_{j}^{(\mathrm{UI})}(t) \beta_{i}^{(\mathrm{U})}+p_{j}^{(\mathrm{AI})}(t) \beta_{i}^{(\mathrm{A})}\right)\right) \gamma=1-\gamma(1+\eta) \beta_{i}^{(\mathrm{U})} \sum_{j} b_{j i} \varepsilon_{j} \\
q_{i}^{(\mathrm{U})}(t) & =\prod_{j}\left(1-b_{j i}\left(p_{j}^{(\mathrm{UI})}(t) \beta_{i}^{(\mathrm{U})}+p_{j}^{(\mathrm{AI})}(t) \beta_{i}^{(\mathrm{A})}\right)\right)=1-(1+\eta) \beta_{i}^{(\mathrm{U})} \sum_{j} b_{j i} \varepsilon_{j}
\end{aligned}
$$

where $\eta=\frac{\beta_{i}^{(\mathrm{A})}}{\beta_{i}^{(\mathrm{U})}}$. According to Eq. (7), the proportion of the infected nodes in the time step $t+1$ is:

$$
\begin{aligned}
& p_{i}^{(\mathrm{I})}(t+1)=1-q_{i}^{(\mathrm{U})}(t)+p_{i}^{(\mathrm{A})}(t)\left[q_{i}^{(\mathrm{U})}(t)-q_{i}^{(\mathrm{A})}(t)\right]+\left[1-\mu_{i}(t)\right] p_{i}^{(\mathrm{I})}(t)= \\
& {\left[1-\mu_{i}(t)\right] p_{i}^{(\mathrm{I})}(t)+\left(p_{i}^{(\mathrm{A})}(t)(\gamma-1)+1\right)(1+\eta) \beta_{i}^{(\mathrm{U})} \sum_{j} b_{j i} \varepsilon_{j}}
\end{aligned}
$$

This formula can be further simplified as:

$$
\sum_{j}\left\{\left\{\left[p_{i}^{(\mathrm{A})}(\gamma-1)+1\right](1+\eta)\right\} b_{j i}-\frac{\mu_{i}}{\beta_{i}^{(\mathrm{U})}} \mathrm{t}_{j i}\right\} \varepsilon_{j}=0
$$

where $t_{j i}$ is the unit matrix. If we let $h_{j i}=\left\{\left[p_{i}^{(\mathrm{A})}(\gamma-1)+1\right](1+\eta)\right\} b_{j i}$ and $\Lambda_{\max }$ be the maximum characteristic values of the matrix, the popular threshold value of model can be described as:

$$
\beta_{i}^{(\mathrm{C})}=\frac{\mu_{i}}{\Lambda_{\max }}
$$

The critical threshold $\beta_{i}{ }^{(\mathrm{C})}$ can be regarded as one solution of the characteristic problem. This threshold is related to the diffusion dynamics of the information layer, especially the parameter $p_{i}{ }^{(\mathrm{A})}$, the ATIS penetration rate, bandwagon effect, and cumulative effect of the information layer. The congestion risk may diffuse to different nodes along the road network if it exceeds the critical threshold.

\section{SIMULATION ANALYSIS AND DISCUSSION}

\subsection{Data acquisition}

The artery network within the fourth ring of Beijing is chosen as the research object in this study. Road situations were investigated, and 12 roads which are prone to traffic congestion were chosen as the initial congestion nodes. Meanwhile, the initial information nodes 1 set to consider the influence of the ATIS penetration rate on the system. Drivers who have ATIS devices are still in State A, and the numerical value of the ATIS penetration rate remains in a fixed proportion with the increase or decrease of commuting flows.

\subsection{Simulation analysis of case study results}

To further study examine and simulate the influence of the ATIS penetration rate, reinforcement effect, and cumulative effect of drivers in the UAU-SIS model on traffic congestion risk propagation, the proportions of congestion nodes under different parameters are proposed. The mean values of all MMCA are higher than 100 operations. When they are not considered independently, the forgetting rate of information, information diffusion probability, transfer probability of the UI node, traffic information warning, cumulative effect, and reinforcement effect are set as 0.3, 0.8, 0.3, 0.5, 0.5 and 0.5, respectively. Each time step in Eq. (6) is iterated by MMCA to obtain the corresponding results. 
The evolution phase diagram of the proportion of congestion nodes in the road network with the ATIS penetration rate and reinforcement effect of drivers when the system reaches the steady state (UI node transfer probability $=0.8$ and traffic information warning $=1$ ) is shown in Fig. 3. The colour scale represents the proportion of congestion. The following outcomes are observed: (1) The proportion of the congestion node is positively related to the UI node transfer probability but negatively related to the ATIS penetration rate and reinforcement effect. When the reinforcement effect is 0.7 or 0.9 , the proportion of the congestion nodes are decreased significantly with the increase of the reinforcement effect. (2) When the traffic information warning is 0.5 , the proportion of the congestion node decreases with the increase of the ATIS penetration rate and reinforcement effect. When the traffic information is 1 , the proportion of the congestion nodes are increased with the increase of the ATIS penetration rate and reinforcement effect. When the reinforcement effect is 0.1 or 0.6 and the ATIS penetration rate is 0.9 or 1 , the proportion of the congestion node drops slightly. When the traffic information warning is 1 and the reinforcement effect is 0.7 or 0.9 , the proportion of the congestion node increases significantly with the increase of the reinforcement effect. In summary, the UI node transfer probability will not change the influence of the ATIS penetration rate and reinforcement effect on the positive trend of congestion risk propagation. The overall influence of increasing the ATIS penetration rate and reinforcement effect on congestion risk propagation is only positive when the traffic information warning is less than 1.
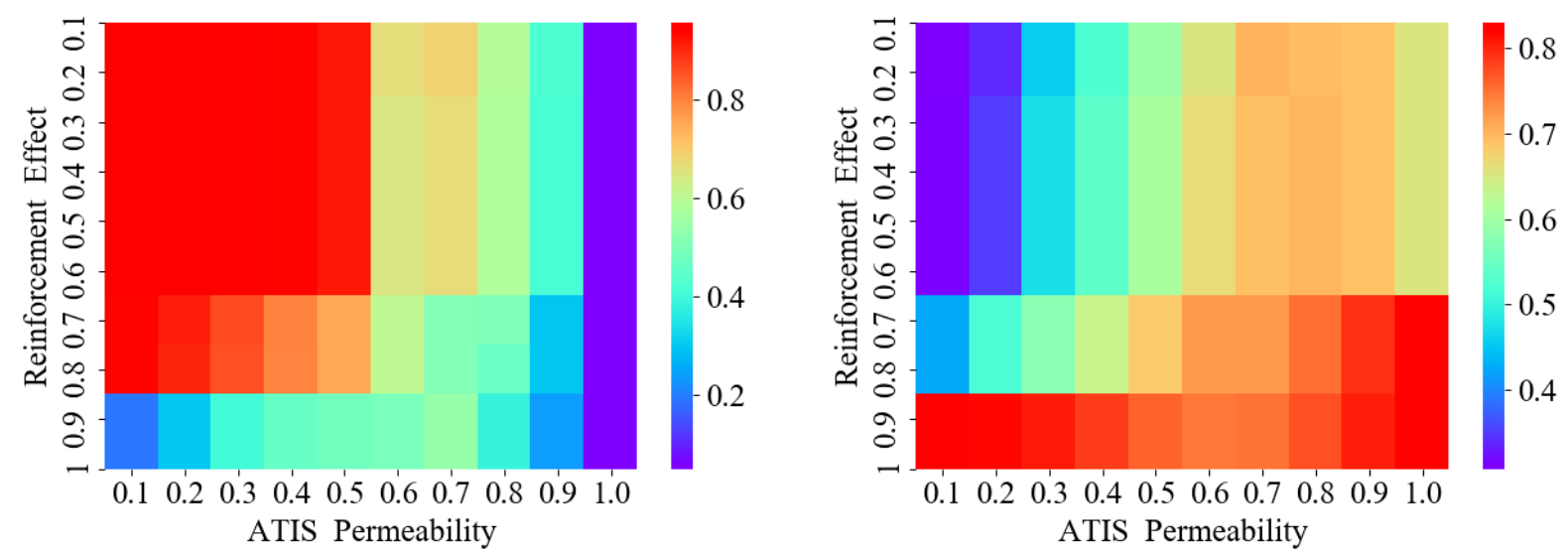

Figure 3: The influence of the ATIS penetration rate, reinforcement effect, UI node transfer probability and traffic information warning on the proportion of congestion nodes.

The evolution phase diagram of the proportion of congestion nodes in the road network with the ATIS penetration rate and reinforcement effect of drivers when the system reaches the steady state (cumulative effect $=0.5$ and 0.8 ) is shown in Fig. 4. The colour scale represents the proportion of congestion. The following outcomes are observed: (1) The influence of the reinforcement effect on system revolution have three obvious interval effects, namely, $(0.1,0.6)$, $(0.7,0.8)$ and $(0.9,1)$. When the reinforcement effects are in the same interval, the influences on system evolution are equal. (2) When the cumulative effect is 0.5 , the proportion of congestion nodes decreases with the increase of the ATIS penetration rate and reinforcement effect. When the reinforcement effect is 0.7 or 0.9 , the proportion of congestion nodes drops significantly with the increase of reinforcement effect. When the cumulative effect is 0.8 , the proportion of congestion nodes are increased with the increase of the ATIS penetration rate and reinforcement effect. When the reinforcement effect is 0.7 or 0.9 , the proportion of congestion nodes are increased dramatically with the increase of the reinforcement effect of drivers. In summary, the influence of traffic information and the reinforcement effect of drivers on congestion risk propagation are determined by the cumulative effect caused by traffic information. 

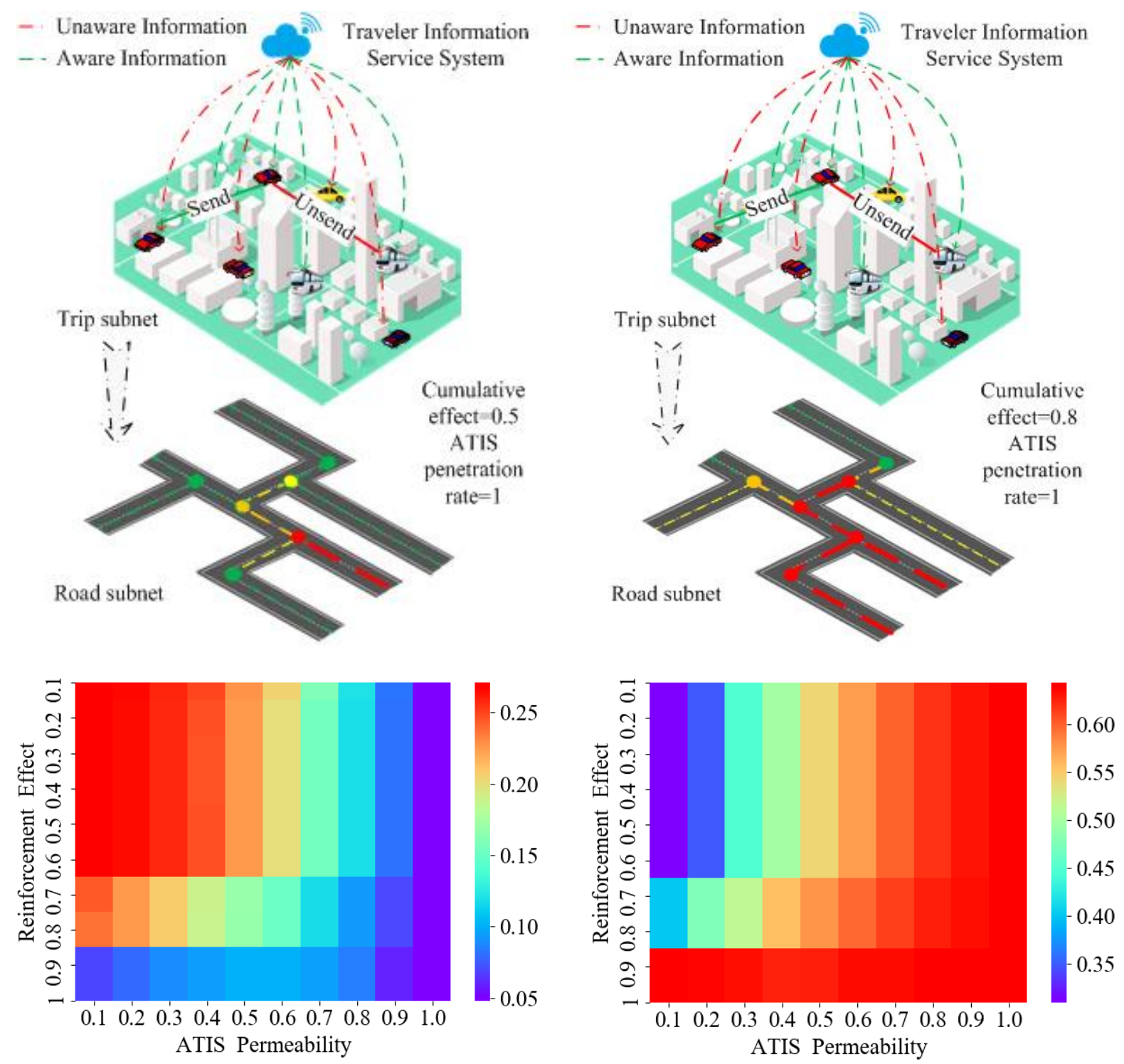

Figure 4: The influence of the ATIS penetration rate, cumulative effect and reinforcement effect on the proportion of congestion nodes.

The evolution phase diagram of the proportion of congestion nodes in the road network with the ATIS penetration rate and the cumulative effect of drivers when the system reaches the steady state (i.e., when the reinforcement effects of the drivers is 0.5 or 1) is shown in Fig. 5. The colour scale represents the proportion of congestion.

The following outcomes are observed: (1) The influence of the cumulative effect on system revolution has three obvious interval effects, namely, $(0.1,0.5),(0.6)$, and $(0.7,1)$. When the cumulative effects are in the same interval, the influences on the system evolution are equal. (2) The proportion of congestion nodes increases with the cumulative effect. When the cumulative effect is 0.6 , the proportion of congestion nodes drops with the increase of the ATIS penetration rate. When the cumulative effect is 0.6 , the proportion of congestion nodes initially increases and then decreases with the increase of the ATIS penetration rate. When the cumulative effect exceeds 0.6 , the proportion of congestion nodes is positively related to the ATIS penetration rate. (3) Given a high ATIS penetration rate and small cumulative effect, the initial proportion of congestion nodes is very low and is lower than its counterpart under a low ATIS penetration rate. With the increase of the cumulative effect, the proportion of congestion nodes increases quickly and finally exceeds that under a low ATIS penetration rate. (4) When 
the reinforcement effect is 0.8 or 1 and the cumulative effect is lower than 0.6 , the proportion of congestion nodes decreases compared to that when the reinforcement effect is 0.5 or 0.3 . The opposite outcome is observed when the cumulative effect exceeds 0.6. In summary, the cumulative effect has a critical value of 0.6. The influence of traffic information and the reinforcement effect on congestion risk propagation is positive when the cumulative effect is lower than 0.6; otherwise, it is positive. When the cumulative effect is lower than 0.6, the proportion of congestion nodes is negatively related to the reinforcement effect. When the cumulative effect is higher than 0.6 , the proportion of congestion nodes is positively related to the reinforcement effect.
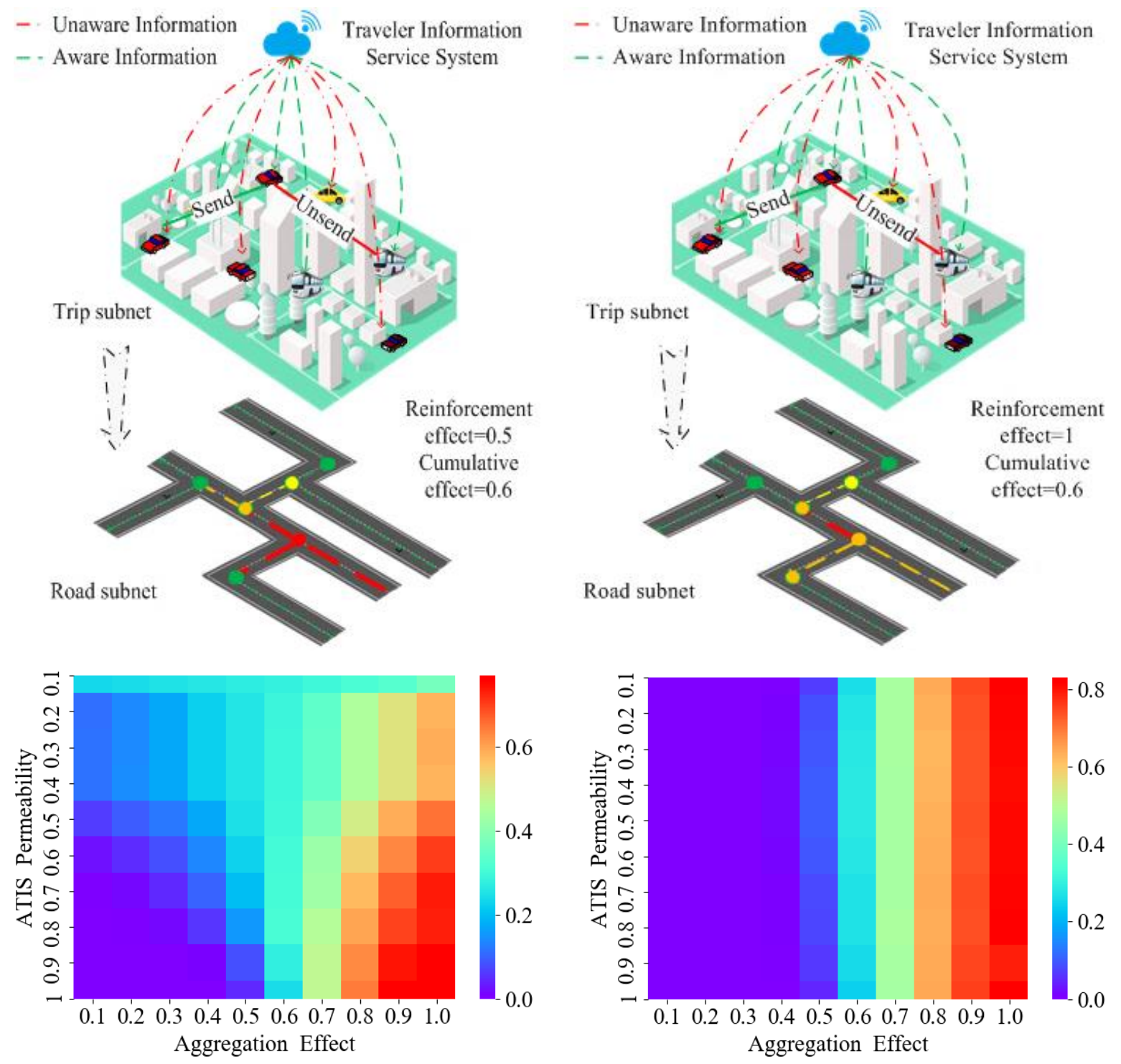

Figure 5: The influence of the ATIS penetration rate, cumulative effect, and reinforcement effect on the proportion of congestion nodes.

\section{CONCLUSION}

To relieve urban traffic congestion, this study investigates the influence of the route choice behaviour of driver groups on the congestion control effect under different market penetration rates of the ATIS. An improved UAU-SIS model that uses a multilayer time-varying network as a framework is constructed and solved by the MMCA. The numerical analysis of the model presents the conclusions: 
(1) The influence of the reinforcement effect on system revolution has three obvious interval effects: from 0.1 to 0.6 , from 0.7 to 0.8 , and from 0.9 to 1 . When the reinforcement effects are in the same interval, the influences on system revolution are equal. At the critical values of 0.7 and 0.9 , the proportion of congestion nodes fluctuates significantly.

(2) The influences of the ATIS penetration rate and reinforcement effect of drivers on congestion risk propagation are determined by the aggregation effect caused by traffic information. Moreover, the aggregation effect has a critical value of 0.6. The influence of the ATIS penetration rate and reinforcement effect on congestion risk propagation is positive when the aggregation effect is below 0.6; otherwise, it is positive. When the aggregation effect is below 0.6 , the proportion of congestion nodes is negatively related to the reinforcement effect.

(3) The UI node transfer probability will not change the influence of the ATIS penetration rate and reinforcement effect on the positive trend of congestion risk propagation. The overall influence of increasing the ATIS penetration rate and reinforcement effect on congestion risk propagation are only positive when the traffic information warning is less than 1 . The aggregation effect caused by traffic information is a key factor that determines the traffic congestion control effect on the basis of traffic information.

This study verifies the influence of drivers' responses to traffic information on the traffic congestion control effect according to the ATIS. Although the drivers' willingness to receive traffic information and the aggregation effect have been considered herein, this work only investigates the influence of the ATIS market penetration rate on traffic information propagation. In reality, the information releases of the ATIS experiences time delays and the traffic information received by drivers might include the wrong route recommendation accordingly. Introducing time parameters into the traffic information propagation process and setting a reasonable time delay are possible research directions in future. Moreover, future studies can incorporate the route choice gaming process among driver groups to further quantify the influence of the psychological characterization of drivers on traffic dispersion.

\section{ACKNOWLEDGEMENT}

The study was supported by the General Projects of the National Social Science Foundation of China (No. 20BGL003).

\section{REFERENCES}

[1] Ben-Elia, E.; Avineri, E. (2015). Response to travel information: a behavioural review, Transport Reviews, Vol. 35, No. 3, 352-377, doi:10.1080/01441647.2015.1015471

[2] Van Essen, M.; Thomas, T.; van Berkum, E.; Chorus, C. (2016). From user equilibrium to system optimum: a literature review on the role of travel information, bounded rationality and non-selfish behaviour at the network and individual levels, Transport Reviews, Vol. 36, No. 4, 527-548, doi:10.1080/01441647.2015.1125399

[3] Hashemi, H.; Abdelghany, K. F. (2016). Real-time traffic network state estimation and prediction with decision support capabilities: Application to integrated corridor management, Transportation Research Part C: Emerging Technologies, Vol. 73, 128-146, doi:10.1016/j.trc.2016.10.012

[4] Kadali, B. R.; Vedagiri, P.; Rathi, N. (2015). Models for pedestrian gap acceptance behaviour analysis at unprotected mid-block crosswalks under mixed traffic conditions, Transportation Research Part F: Traffic Psychology and Behaviour, Vol. 32, 114-126, doi:10.1016/ j.trf.2015.05.006

[5] Shen, J.; Yang, G. (2020). Integrated empirical analysis of the effect of variable message sign on driver route choice behaviour, Journal of Transportation Engineering, Part A: Systems, Vol. 146, No. 2, Paper 04019064, 9 pages, doi:10.1061/JTEPBS.0000295

[6] Suryani, E.; Hendrawan, R. A.; Adipraja, P. F. E.; Indraswari, R. (2020). System dynamics simulation model for urban transportation planning: a case study, International Journal of Simulation Modelling, Vol. 19, No. 1, 5-16, doi:10.2507/IJSIMM19-1-493 
[7] Dai, R.; Lu, Y.; Ding, C.; Lu, G.; Wang, Y. (2019). A simulation-based approach to investigate the driver route choice behaviour under the connected vehicle environment, Transportation Research Part F: Traffic Psychology and Behaviour, Vol. 65, 548-563, doi:10.1016/j.trf.2018.04.008

[8] Diop, E. B.; Zhao, S.; Tran, V. D. (2020). Modeling travelers' acceptance of variable message signs: a hierarchical hybrid choice model, Journal of Transportation Engineering, Part A: Systems, Vol. 146, No. 12, Paper 04020134, 14 pages, doi:10.1061/jtepbs.0000456

[9] Khoo, H. L.; Asitha, K. S. (2016). An impact analysis of traffic image information system on driver travel choice, Transportation Research Part A: Policy and Practice, Vol. 88, 175-194, doi:10.1016/j.tra.2016.03.014

[10] Kos, G.; Ivandic, N.; Vidovic, K. (2020). Tourism as a factor of demand in public road passenger transportation in the Republic of Croatia, Technical Journal, Vol. 14, No. 1, 76-87, doi:10.31803/tg-20191210120738

[11] Klein, I.; Ben-Elia, E. (2018). Emergence of cooperative route-choice: A model and experiment of compliance with system-optimal ATIS, Transportation Research Part F: Traffic Psychology and Behaviour, Vol. 59, Part A, 348-364, doi:10.1016/j.trf.2018.09.007

[12] Yu, Y.; Han, K.; Ochieng, W. (2020). Day-to-day dynamic traffic assignment with imperfect information, bounded rationality and information sharing, Transportation Research Part C: Emerging Technologies, Vol. 114, 59-83, doi:10.1016/j.trc.2020.02.004

[13] Marti-Calatayud, P.; Romero-Chavarro, J.; Montagud-Aguar, M.; Arcos-Usero, L.; Garcia-Pellicer, M.; Quijano-Lopez, A. (2021). Three-level methodology for secure and efficient grid integration of electric vehicle, Dyna, Vol. 96, No. 3, 264-269, doi:10.6036/10013

[14] Lu, Q.; Tettamanti, T. (2021). Impacts of connected and automated vehicles on freeway with increased speed limit, International Journal of Simulation Modelling, Vol. 20, No. 3, 453-464, doi:10.2507/IJSIMM20-3-556

[15] Li, M. M.; Lu, J.; Sun, J. H. (2020). Day-to-day traffic assignment model with multiple types of information, Journal of Transportation Systems Engineering and Information Technology, Vol. 20, No. 4, 97-105, doi:10.16097/j.cnki.1009-6744.2020.04.015

[16] Zheng, C. Y.; Xia, C. Y.; Guo, Q. T.; Dehamer, M. (2018). Interplay between SIR-based disease spreading and awareness diffusion on multiplex networks, Journal of Parallel and Distributed Computing, Vol. 115, 20-28, doi:10.1016/j.jpdc.2018.01.001

[17] Wang, Z.; Guo, Q.; Sun, S.; Xia, C. (2019). The impact of awareness diffusion on SIR-like epidemics in multiplex networks, Applied Mathematics and Computation, Vol. 349, 134-147, doi:10.1016/j.amc.2018.12.045

[18] Huo, L.; Guo, H.; Cheng, Y.; Xie, X. (2020). A new model for supply chain risk propagation considering herd mentality and risk preference under warning information on multiplex networks, Physica A: Statistical Mechanics and its Applications, Vol. 545, Paper 123506, 11 pages, doi:10.1016/j.physa.2019.123506

[19] Xiao, Y.; Zhang, L.; Li, Q.; Liu, L. (2019). MM-SIS: Model for multiple information spreading in multiplex network, Physica A: Statistical Mechanics and its Applications, Vol. 513, 135-146, doi:10.1016/j.physa.2018.08.169 